\title{
Global existence and estimates of the solutions to nonlinear integral equations
}

\author{
Alexander G. Ramm $^{1 *}$ \\ ${ }^{1}$ Department of Mathematics, Kansas State University, , Manhattan, KS 66506, USA, http://www.math.ksu.edur ramm \\ ${ }^{*}$ Corresponding author E-mail: ramm@math.ksu.edu
}

\begin{abstract}
It is proved that a class of nonlinear integral equations of the Volterra-Hammerstein type has a global solution, that is, solutions defined for all $t \geq 0$, and estimates of these solutions as $t \rightarrow \infty$ are obtained. The argument uses a nonlinear differential inequality which was proved by the author and has broad applications.
\end{abstract}

Keywords: nonlinear integral equations

\section{Introduction}

Consider the equation:

$u(t)=\int_{0}^{t} e^{-a(t-s)} h(u(s)) d s+f(t):=T(u), \quad t \geq 0 ; \quad a=$ const $>0$.

that is, Volterra-Hammerstein equation. There is a large literature on nonlinear integral equations,[1], [6]. The usual methods to study such equations include fixed-point theorems such as contraction mapping principle and degree theory, (Schauder and Leray-Schauder theorems). The goal of this paper is to give a new approach to a study of equation (1). We give sufficient conditions for the global existence of solutions to (1) and their estimates as $t \rightarrow \infty$.

Denote $f^{\prime}:=\frac{d f}{d t}$. By $c>0$ various constants will be denoted.

Let us formulate our assumptions:

$|h(u)| \leq c|u|^{b}, \quad\left|h^{\prime}(u)\right| \leq c|u|^{b-1}, \quad b \geq 2$,

$|f(t)|+a\left|f^{\prime}(t)\right| \leq c e^{-a_{1} t}, \quad a_{1}=$ const $>0$.

Our approach is based on the author's results on the nonlinear differential inequality formulated in Theorem 1 (see [2]-[5]). These results have been used by the author in a study of stability of solutions to abstract nonlinear evolution problems ([5]).

Denote $\mathbb{R}_{+}=[0, \infty)$.

Theorem 1. Let $g \geq 0$ solve the inequality

$g^{\prime}(t) \leq-a g(t)+\alpha(t, g)+\beta(t), \quad t \geq 0, \quad a=$ const $>0$,

where $\alpha(t, g) \geq 0$ and $\beta(t) \geq 0$ are continuous functions of $t, t \in \mathbb{R}_{+}$ and $\alpha(t, g)$ is locally Lipschitz, with respect to $g$. If there exists a function $\mu(t)>0$, defined on $\mathbb{R}_{+}, \mu \in C^{1}\left(\mathbb{R}_{+}\right)$, such that

$\alpha\left(t, \frac{1}{\mu(t)}\right)+\beta(t) \leq \frac{1}{\mu(t)}\left(a-\frac{\mu^{\prime}(t)}{\mu(t)}\right), \quad \forall t \geq 0$, and

$g(0) \mu(0) \leq 1$,

then $g$ exists on $\mathbb{R}_{+}$and

$0 \leq g(t) \leq \frac{1}{\mu(t)}, \quad \forall t \geq 0$.

A proof of Theorem 1 can be found in [5]. Its idea is described in Section 2.

The result of this paper is formulated in Theorem 2 .

Theorem 2. Assume that (2) and (3) hold, $a \geq 2, b \geq 2, c \in(0,0.75)$, $p \in\left(0, \min \left(0.75 a, a_{1}\right)\right), R=(b-1)^{1 / b}$. Then any solution to $(1)$ exists on $\mathbb{R}_{+}$and satisfies the estimate

$|u(t)| \leq R^{-1} e^{-p t}, \quad \forall t \geq 0, \quad p \in\left(0, \min \left(0.25 a_{1}, a\right)\right)$.

In Section 2 Theorem 2 is proved.

\section{Proof of Theorem 2}

Let us reduce equation (1) to the form suitable for an application of Theorem 1. Differentiate (1) and get

$u^{\prime}=f^{\prime}-a \int_{0}^{t} e^{-a(t-s)} h(u(s)) d s+h(u(t))$.

Let $g(t):=|u(t)|$ and take into account that $|F(t)| \leq c e^{a_{1} t}, F:=$ $f^{\prime}+a f$.

From (1) one gets $\int_{0}^{t} e^{-a(t-s)} h(u(s)) d s=u-f$. This and equation (9) imply

$u^{\prime}=f^{\prime}-a(u-f)+h(u(t))$.

Therefore, one gets

$u^{\prime}=-a u+h(u)+F, \quad F:=f^{\prime}+a f$. 
Multiply (11) by $\bar{u}$, where $\bar{u}$ stands for complex conjugate of $u$, and get

$u^{\prime} \bar{u}=-a g^{2}+h(u) \bar{u}+F \bar{u}$.

One has

$u^{\prime} \bar{u}+u(\bar{u})^{\prime}=\frac{d g^{2}}{d t}=2 g g^{\prime}$.

We define the derivative as $g^{\prime}=\lim _{h \rightarrow+0} \frac{g(t+h)-g(t)}{h}$. With this definition, $g(t)$ is differentiable at every point if $u(t)$ is continuously differentiable for all $t \geq 0$. Any solution $u(t)$ to (1) is continuously differentiable under our assumptions. Take complex conjugate of (12), add the resulting equation to (12) and take into account (13). This yields

$2 g g^{\prime}=-2 a g^{2}+2 \operatorname{Re}(h(u) \bar{u})+2 \operatorname{Re}(F \bar{u})$.

Since $g \geq 0$, one derives from (14), using assumptions (2) and (3), that

$g^{\prime}(t) \leq-a g(t)+c g^{b}+c e^{-a_{1} t}$.

Let

$\mu(t)=R e^{p t}, \quad R=$ const $>0, \quad p \in\left(0, \min \left(0.25 a, a_{1}\right)\right)$.

Condition (5) can be written as

$\frac{c}{R^{b} e^{b p t}}+c e^{-a_{1} t} \leq \frac{1}{R e^{p t}}(a-p), \quad t \in \mathbb{R}_{+}$.

This inequality holds if

$\frac{c}{R^{b-1} e^{(b-1) p t}}+c R e^{-\left(a_{1}-p\right) t} \leq \frac{3 a}{4}, \quad t \in \mathbb{R}_{+}$.

Inequality (18) holds if $b \geq 1$ and

$\frac{1}{R^{b-1}}+R \leq \frac{3 a}{4 c}$.

The minimum of the left side of (19) is attained at $R=(b-1)^{1 / b}$ and is equal to $\frac{b}{(b-1)^{(b-1) / b}}$. Thus, (19) holds if

$\frac{b}{(b-1)^{(b-1) / b}} \leq \frac{3 a}{4 c}$.

For example, assume that

$$
a \geq 2, \quad c \leq 0.75 .
$$

Then (20) holds if $b \leq 2(b-1)^{(b-1) / b}$, that is, if

$b^{b} \leq 2^{b}(b-1)^{b-1}$.

Inequality (21) holds if $b \geq 2$. Thus, by Theorem1, any solution $u(t)$ of (1) exists globally and

$|u(t)| \leq \frac{e^{-p t}}{R}$,

provided that

$$
\begin{array}{r}
|u(0)| R \leq 1, \quad R=(b-1)^{1 / b}, \quad a \geq 2, \quad b \geq 2, \quad c=0.75, \\
p \in\left(0, \min \left(0.25 a, a_{1}\right)\right) .
\end{array}
$$

Inequality $|u(0)| R \leq 1$ holds if $|f(0)| R \leq 1$. By assumption (3) this inequality holds if $c \leq \frac{1}{R}$. Theorem 2 is proved.

Let us prove existence of a solution to (1) using the contraction mapping principle and Theorem 2.

By estimate (22) one has $|u(t)| \leq \frac{1}{R}$ for all $t \geq 0$. Therefore, using assumptions (2) and (3), one gets

$|T u| \leq c+\frac{c}{a R^{b}} \leq \frac{1}{R}$, provided that $c R \leq \frac{1}{1+\frac{1}{a R^{b}}}$. For $R=(b-1)^{1 / b}$ this inequality holds if $c$ is sufficiently small. If (24) holds, then $T$ maps the ball $B_{R}:=$ $\left\{u:\|u\| \leq \frac{1}{R}\right\}$ into itself. Here $\|u\|=\max _{t \geq 0}|u(t)|$.

On the ball $B_{R}$ the operator $T$ is a contraction:

$\|T u-T v\| \leq\left\|\int_{0}^{t} e^{-a(t-s)} c\left|\eta^{b-1}\right| d s\left|\|\mid u-v\| \leq \frac{c}{R^{b-1} a}\|u-v\|\right.\right.$,

where the assumption (2) was used, and $\eta$ is the "middle" element between $u$ and $v,\|\eta\| \leq \frac{1}{R}$. The integral in (25) is estimated as follows:

$\| \int_{0}^{t} e^{-a(t-s)} c\left|\eta^{b-1}\right| d s|| \leq \frac{c}{R^{b-1}} \max _{t \geq 0} \int_{0}^{t} e^{-a(t-s)} d s \leq \frac{c}{R^{b-1} a}$.

If

$\frac{c}{R^{b-1} a}<1$,

then $T$ is a contraction on $B_{R}$. Condition (27) holds if $c$ is sufficiently small. Thus, if condition (27) and the assumptions of Theorem 2 hold, then, by the contraction mapping principle, there exists a unique solution to (1) in the ball $B_{R}$.

For convenience of the reader we sketch the idea of the proof of Theorem 1 following [2]-[5].

Inequality (5) can be written for the function $w=\frac{1}{\mu}$ as follows:

$-a w+\alpha(t, w)+\beta(t) \leq w^{\prime}$.

From (4) and (28) by a comparison lemma for ordinary differential equations it follows that

$0 \leq g(t) \leq \frac{1}{\mu(t)}$

provided that $g(0) \leq w(0)=\frac{1}{\mu(0)}$. The last inequality is the assumption (6). Since $\mu(t)>0$ and is assumed to be defined for all $t \geq 0$, the function $w=\frac{1}{\mu}$ is defined for all $t \geq 0$. Since $0 \leq g(t) \leq \frac{1}{\mu(t)}$, and $g(t):=|u(t)|$, the function $u$ is defined for all $t \geq 0$.

If $\lim _{t \rightarrow \infty} \mu(t)=\infty$, then $\lim _{t \rightarrow \infty}|u(t)|=0$ by estimate (29).

\section{References}

[1] K. Deimling, Nonlinear functional analysis, Springer-Verlag, Berlin, 1985

[2] A.G.Ramm, Asymptotic stability of solutions to abstract differential equations, Journ. of Abstract Diff. Equations and Applications (JADEA), 1, N1, (2010), 27-34.

[3] A.G.Ramm, A nonlinear inequality and evolution problems, Journ, Ineq. and Special Funct., (JIASF), 1, N1, (2010), 1-9.

[4] A.G.Ramm, Stability of solutions to some evolution problems, Chaotic Modeling and Simulation (CMSIM), 1, (2011), 17-27.

[5] A.G.Ramm, Large-time behavior of solutions to evolution equations, in Handbook of Applications of Chaos Theory, Chapman and Hall/CRC, (ed. C.Skiadas), pp. 183-200.

[6] P. Zabreiko et al, Integral equations: a reference text, Leyden, Noordhoff International Pub., 1975. 